\title{
Potencial das microalgas na indústria farmacêutica
}

Juliana Mesadri, Roger Wagner, Mariane Bittencourt Fagundes

https://doi.org/10.4322/mp.978-65-994457-8-1.c2

\section{Resumo}

As microalgas são consideradas fontes confiáveis de compostos bioquimicamente ativos a serem potencialmente utilizadas como matéria-prima pela indústria farmacêutica. As microalgas possuem uma biomassa com elevada biodiversidade e variabilidade de composição química, além de elevada produtividade, de forma que também são conhecidas por serem fontes sustentáveis e renováveis. Dentre as espécies de microalgas, a Chlorella sp. é caracterizada por ser uma cepa eucariótica, e a Spirulina sp. como uma cepa procariótica, sendo atualmente destacada no mercado da indústria farmacêutica em termos de produção de compostos bioativos. Dentre os inúmeros compostos passíveis de serem extraídos, que apresentam potencial de exploração comercial estão os carotenoides, ácidos graxos poli-insaturados, ficobilinas, vitaminas, esteróis, polissacarídeos, e entre outros que podem ser estudados em sinergia, ou não. Desta forma, estes metabólitos podem estar atribuídos a atividades antibacterianas, antitumorais, antifúngicas e antivirais. Muitos compostos não convencionais das microalgas, são difíceis de sintetizar, logo o uso das biomassas permite a obtenção de substâncias de forma eficiente como precursores no desenvolvimento de fármacos. Os seus produtos são benéficos não apenas para o setor farmacêutico, mas também para a indústria de alimentos. Existem vários pontos da indústria em que as microalgas podem ser utilizadas, podendo destacar também o seguimento de suplemento alimentar, cosméticos e também podem ser utilizadas para a remediação e redução de tóxicos nas águas das indústrias farmacêuticas. Desse modo as microalgas, tanto seus extratos, quanto seus compostos isolados, podem ser utilizados nas diferentes formulações farmacêuticas.

Palavras-chave: biotecnologia, compostos bioativos, indústria farmacêutica, microalgas. 


\section{Introdução}

As algas constituem um grupo de organismos com uma ampla diversidade morfológica, assim, cada espécie pode desempenhar diferentes funções. Dentre eles, se destacam as microalgas, que são micro-organismos fotossintéticos, classificados como unicelulares, ainda podem ser designadas em dois grupos estruturais: procarióticas, também conhecidas como as cianobactérias, e em eucarióticas, que englobam representantes nas divisões Chlorophyta, Euglenophyta, Rhodophyta, Haptophyta (Prymnesiophyta), Heterokontophyta (Bacillariophyceae, Chrysophyceae, Xantophyceae etc.), Cryptophyta e Dinophyta. (BICUDO e MENEZES, 2010; DERNER et al., 2006). Ambos os grupos são considerados fontes potenciais de energia, combustível, alimentos e outros produtos de grande valor para a química fina (GARCIA et al., 2017).

Existem mais de cinquenta mil tipos diferentes de espécies de microalgas presentes nos oceanos e na água doce (lagos, lagoas e rios), dentre as quais cerca de trinta mil já foram estudadas. As células microalgais podem variar de 0,2 a $2 \mu \mathrm{m}$ e, quando se apresentam em formas filamentosas com tamanhos de $100 \mu \mathrm{m}$ ou superiores. A maior parte dos estudos está associada com o uso das biomassas microalgais aplicadas em alimentos, sendo explorado há milhares de anos. Além disso as microalgas possuem a habilidade de converter energia solar em energia química fixando $\mathrm{CO}_{2}$ (BULE et al., 2018; FORZZA, 2010).

Em termos econômicos, embora já exista a aplicabilidade de algumas cepas no mercado, a produção comercial de microalgas é de aproximadamente 5.000 toneladas por ano de matéria seca. A maior parte da biomassa produzida é associada a alimentação, sendo cerca de nove décimos do cultivo de algas estão localizados na Ásia (BULE et al., 2018). Assim, a exploração de microalgas para a produção ambiental e industrial de produtos naturais acaba por se tornar um grande setor em expansão.

\section{Produção de compostos de interesse}

Ao longo da história, as microalgas são empregadas para diversas finalidades, sendo a mais antiga, sua utilização para alimentação de humanos e animais (SIMÕES et al., 2016). Atualmente, muitas microalgas cultivadas em condições específicas estão sendo vistas como alternativas viáveis para a produção de uma série de compostos nutracêuticos (GARCIA et al., 2017). 
Esses organismos têm potencial para coprodução de outras moléculas como pigmentos, proteínas, ácidos graxos poli-insaturados, e outros antioxidantes. Assim, as microalgas estão ganhando atenção como agentes terapêuticos para inúmeros distúrbios de saúde e outras aplicações nas indústrias alimentícia, cosmética e farmacêutica (BULE et al., 2018). Ademais, as microalgas também podem sobreviver em condições adversas, ou seja, resistir ao calor, frio, radiação ultravioleta, salinidade e dessecação, devido à sua capacidade de se adaptar a mudanças fisiológicas, produzindo substâncias tolerantes ao estresse (PANGESTUTI et al., 2018).

Os primeiros produtos comerciais provenientes de algas foram os polissacarídeos extraídos de macroalgas e utilizados como fonte de ficocolóides (ágar, carragenina e alginato). As microalgas, contudo, só entraram no mercado, para este tipo de aplicação no final do século passado, quando a tecnologia de cultivo deste organismo unicelular foi desenvolvida. Quatro espécies de microalgas (Spirulina, Chlorella, Dunaliella e Nannochloropsis) atingiram o nível de produção em cultivo em larga escala aberto, enquanto que mais algumas espécies unicelulares foram ampliadas com sucesso em sistema de cultivo realizados em biorreatores fechados (BEN-AMOTZ, 2009).

Existem quatro principais tipos de técnicas de cultivo de microalgas: fotoautotrófico, heterotrófico, mixotrófico e fotoheterotrófico. Com base nessas técnicas de cultivo, novas tecnologias foram desenvolvidas para o cultivo microalgal as quais são atualmente aplicadas na produção de biomassa algal: tanques abertos, fotobiorreatores fechados e reatores de fermentação. Dentre os sistemas abertos, a utilização dos tanques rasos abertos se destaca pela quantidade de produção, onde o cultivo realizado pode variar de centenas a milhas de metros quadrados. No entanto, alguns inconvenientes deste tipo de cultivo ocorrem pela possibilidade de contaminação por outros organismos e/ou por outras espécies de microalgas. Desenvolvidos mais recentemente e tecnologicamente mais avançados, os sistemas fechados são mais adequados, como os fotobiorreatores, eles possuem as melhores condições para a produção de praticamente todas as espécies de microalgas, protegendo a cultura da contaminação de outros organismos e permitindo o controle das condições do cultivo (quantidade dos nutrientes, temperatura, iluminação, pH etc.), oferecendo melhor qualidade da biomassa gerada. Estes fotobiorreatores são planos ou 
tubulares e podem adotar uma variedade de modelos e modos de operação, embora apresentem um maior custo de construção e operação em comparação aos sistemas abertos. Uma combinação de fotobiorreatores e tanques acessíveis vem sendo definidos como uma eficiente alternativa para maiores produtividades e custos relativamente acessíveis na produção das microalgas (DANTAS, 2013; DERNER et al., 2006; SIMÕES et al., 2016).

A biotecnologia microalgal ganhou importância nas últimas décadas e as suas aplicações variam da simples produção de biomassa para alimentação, como já comercial, a valiosos produtos para aplicações farmacêuticas. Para a maioria das aplicações, o mercado ainda está em desenvolvimento e seu uso se estendendo a novas áreas. Os avanços moleculares genéticos nas microalgas também têm um impacto profundo sobre o desenvolvimento de processos e tecnologias (SIMÕES et al., 2016).

Com uma enorme biodiversidade de microalgas e recentes desenvolvimentos em engenharia genética, este grupo de organismos representa uma das fontes mais promissoras para novos ingredientes passíveis de serem aplicados em diversos produtos. Além disso, espera-se que esses produtos se tornem muito competitivos no mercado devido ao seu maior valor biológico, melhoria no processo de cultivo e menor custo de produção do que os produtos sintéticos (THIYAGARASAIYAR et al., 2020).

\section{Compostos bioativos microalgais}

Existem milhares de compostos bioativos distribuídos em diferentes grupos químicos, os quais normalmente são metabólitos secundários, produzidos em pequenas quantidades principalmente pelo sistema de defesa. Por existirem milhares de biomoléculas, as mesmas podem apresentar diferentes funções metabólicas. As microalgas são uma rica fonte de compostos com atividade, os quais dentre estes destacam-se: os ácidos graxos da família ômega-3, carotenoides, fitosteróis, terpenos, compostos fenólicos e amino ácidos (PIMENTEL et al., 2019).

Os compostos bioativos presentes em alimentos funcionais têm como local de ação alvos fisiológicos específicos, modulando a defesa antioxidante e de processos inflamatórios e mutagênicos (PIMENTEL et al., 2019). A diversidade de compostos em microalgas chega a ser 10 vezes maior que em 
plantas terrestres, e isto está associado à sua sobrevivência em condições adversas. A Tabela 1 apresenta os principais bioativos provenientes de microalgas no mercado e suas aplicações.

Tabela 1. Principais compostos bioativos no mercado, provenientes da biotecnologia microalgal.

\begin{tabular}{|c|c|c|}
\hline & Produtos & $\begin{array}{c}\text { Principais } \\
\text { Aplicações }\end{array}$ \\
\hline Biomassa & Biomassa & $\begin{array}{l}\text { Alimentos funcionais } \\
\text { Alimentos naturais } \\
\text { Aquicultura } \\
\text { Condicionador do solo }\end{array}$ \\
\hline $\begin{array}{c}\text { Compostos } \\
\text { Fenólicos }\end{array}$ & $\begin{array}{l}\text { Ácido Benzoico } \\
\text { Ácido Cinâmico } \\
\text { Polifenóis }\end{array}$ & $\begin{array}{l}\text { Aditivos Alimentares } \\
\text { Cosméticos } \\
\text { Corantes }\end{array}$ \\
\hline Pigmentos & $\begin{array}{l}\text { Carotenos } \\
\text { Xantofilas } \\
\text { Ficobilinas } \\
\text { Clorofilas } \\
\end{array}$ & $\begin{array}{l}\text { Aditivos Alimentares } \\
\text { Cosméticos } \\
\text { Corantes }\end{array}$ \\
\hline Alcaloides & $\begin{array}{l}\text { Feniletilamina } \\
\text { Tiramina }\end{array}$ & $\begin{array}{l}\text { Fármacos } \\
\text { Pesquisa }\end{array}$ \\
\hline $\begin{array}{c}\text { Vitaminas e } \\
\text { Minerais }\end{array}$ & $\begin{array}{l}\text { Vitamina B1, B2, B6, niacina, folato, } \\
\text { biotina, ácido pantotênico, C, D2, E e K } \\
\text { Mineral potássio, cálcio, magnésio e } \\
\text { selênio }\end{array}$ & Aditivos Alimentares \\
\hline $\begin{array}{l}\text { Ácidos } \\
\text { Graxos }\end{array}$ & $\begin{array}{l}\text { Ácido araquidónico - ARA } \\
\text { Ácido eicosapentaenóico - EPA } \\
\text { Ácido docosahexaenóico - DHA } \\
\text { Ácido gama-linolénico - GLA } \\
\text { Ácido linoléico - LA }\end{array}$ & Aditivos Alimentares \\
\hline Enzimas & $\begin{array}{l}\text { Superóxido dismutase - SOD } \\
\text { Fosfoglicerato quinase - PGK } \\
\text { Luciferase e Luciferina } \\
\text { Enzimas de restrição }\end{array}$ & $\begin{array}{l}\text { Pesquisa } \\
\text { Medicina } \\
\text { Fármacos }\end{array}$ \\
\hline Polímeros & $\begin{array}{l}\text { Polissacarídeos } \\
\text { Amido } \\
\text { Ácido poli- } \beta \text {-hidroxibutírico - PHB } \\
\text { Peptídeos } \\
\text { Toxinas }\end{array}$ & $\begin{array}{l}\text { Aditivos Alimentares } \\
\text { Cosméticos } \\
\text { Fármacos }\end{array}$ \\
\hline $\begin{array}{l}\text { Produtos } \\
\text { Especiais }\end{array}$ & $\begin{array}{l}\text { Prolina } \\
\text { Arginina } \\
\text { Ácido aspártico } \\
\text { Esteróis }\end{array}$ & $\begin{array}{l}\text { Aditivos Alimentares } \\
\text { Fármacos } \\
\text { Pesquisa }\end{array}$ \\
\hline
\end{tabular}

Fonte: Fonseca J. A., 2016; Gago A. S., 2016. 
Com relação as moléculas lipídicas, os maiores estudos e aplicações estão associados aos ácidos graxos insaturados como ácido eicopentaenoico (EPA), $\alpha$-linolêico (GLA), ácido araquidônico (ARA), e ácido docosaexaenoico (DHA). O uso de EPA ocorre em nutracêuticos, e anti-inflamatório. GLA e ARA possuem maior aplicação para problemas associados a vasoconstrição ocasionado por plaquetas, já o ácido DHA possui ampla aplicação como nutracêutico para crianças, pois está associado a auxílio no desenvolvimento cerebral (KUMAR et al., 2019).

As vitaminas são outra importante classe de moléculas que vem sendo aplicada no mercado sendo que muitos destes compostos amplamente encontrados em microalgas, dentre estes os principais são: vitamina $\mathrm{C}, \mathrm{K}, \mathrm{B} 12$, A e $\alpha$-tocopherol. O principal uso das vitaminas no mercado é como antioxidante, auxiliando no sistema imune, no entanto, outras inúmeras aplicações essas moléculas podem apresentar, como seu uso em probióticos como cofatores enzimáticos e outros (DUFOSSÉ, 2016).

Os pigmentos microalgais são consideradas moléculas bioativas de elevado interesse, dentre eles o carotenóide astaxantina destaca-se em produção pela microalga Haematococcus pluvialis, a qual também produz $\beta$ caroteno, ficocianina e ficoeritrina (FAGUNDES et al., 2019). A luteína, zeaxantina e cantaxantina são carotenoides utilizados em indústrias farmacêuticas e podem provir de microalgas.

Os esteróis, são considerados metabólitos secundários microalgais e moléculas que possuem como precursor metabólico o esqualeno as cepas possuem habilidade de produzir tanto os fitoesteróis, como: stigmasterol e $\beta$ sitosterol, quanto compostos da classe dos zoosterois como: lanoesterol e colesterol (FAGUNDES et al., 2019). No mercado atual os compostos mais explorados, são: Brassicaesterol e stigmasterol. Essas moléculas têm sido reportadas em estudos devido sua ação hipocolesterolêmica (RANDHIR et al., 2020), entre outros. Além disso, muitas pesquisas relataram que outros metabólitos secundários derivados de microalgas, como fucoidan, fucoxantina, polissacarídeo sulfatado, polifenol e fucosterol demonstram efeitos antiinflamatórios, antioxidantes, anticâncer e antibacterianos (THIYAGARASAIYAR et al., 2020). 
Estes são alguns dos principais ativos provenientes de cepas microalgais, sendo que vários outros ativos podem ser explorados e serão abordados nos tópicos abaixo.

\section{Principais aplicabilidades dos metabólitos microalgais}

Muitos compostos não convencionais das microalgas, são difíceis de serem sintetizados quimicamente, então o uso dessas biomassas como fonte dessas biomoléculas de forma direta e eficiente. As microalgas podem ser utilizadas em vários pontos da indústria, pois elas possuem potencial não apenas para fármacos e cosméticos, mas também para o seguimento de suplementação alimentar. Ou seja, seus produtos são benéficos para setor farmacêutico e também para a indústria de alimentos (DERNER, 2006; PIMENTEL, 2019).

O termo "nutracêutico" foi criado em 1989 pela Fundação para Inovação em Medicina (Nova York, EUA). Logo, um nutracêutico é considerado um alimento funcional, pois de forma geral, entende-se que aqueles alimentos que contêm um ou mais compostos com impacto positivo na saúde, conhecidos como substâncias bioativas, possuem ação metabólica ou fisiológica específica no organismo humano (PEDROSA, 2015). Estes compostos podem variar de nutrientes isolados, suplementos dietéticos e dietas a alimentos "projetados" geneticamente modificados, produtos fitoterápicos e produtos processados, como cereais, sopas e bebidas (BISSON, 2020). A legislação brasileira não reconhece oficialmente o termo nutracêuticos, entretanto, define substância bioativa, que é a definição oficial mais compatível (PEDROSA, 2015).

As autoridades de saúde pública consideram a prevenção e o tratamento com produtos nutracêuticos um poderoso instrumento para manter a saúde e agir contra doenças agudas e crônicas induzidas nutricionalmente, promovendo assim saúde, longevidade e qualidade de vida ideais (BISSON, 2020). Enquanto os nutracêuticos são feitos a partir de alimentos ou de parte de um alimento, os suplementos alimentares, constituem outra categoria de alimentos funcionais, as quais são substâncias isoladas usadas sozinhas ou em misturas com o objetivo de adicionar micronutrientes quando o corpo necessita delas (PIMENTEL et al., 2019). Os nutracêuticos extraídos de fontes vegetais (fitocomplexos) ou que são o complexo metabólito ativo (se de origem animal) devem ser entendidos como um conjunto de substâncias farmacologicamente ativas com propriedades 
terapêuticas inerentes devido a seus princípios ativos naturais de eficácia reconhecida. Devem ser administrados na forma farmacêutica apropriada (p. ex., cápsulas, comprimidos, soluções, xaropes etc.) (BISSON, 2020).

Nesse sentido mercado de nutracêuticos de microalgas é dominado por duas cianobactérias, universalmente conhecidas como Spirulina e Klamath, além da espécie clorofícea Chlorella. Hoje em dia, a Spirulina é utilizada em suplementos alimentares, na forma de comprimidos, isoladamente ou em associação com outras algas ou extratos de plantas, para uso humano ou animal (VIEIRA et al., 2020).

A Spirulina é considerada uma boa fonte de diversos nutrientes, apresentando um teor de proteína alto com uma composição aminoacídica balanceada, tornando-a desejável como um suplemento alimentar. Adicionalmente, a biomassa dessa microalga apresenta um teor de óleo elevado e alta qualidade, apresentando os ácidos graxos $\alpha$-linolênico ( $A L A)$, linoléico (LA), ácido estearidônico (SDA), EPA, DHA e AA. Além disso, o seu conteúdo em vitaminas, como as do grupo $B$, principalmente a $B 12$, e a vitamina $E$, é considerado relevante e completo. A Spirulina também é valorizada pela presença de diversos minerais, como potássio, cálcio, magnésio e selênio (GROSSHAGAUER et al., 2020; NICOLETTI, 2016).

A Chlorella possui uma grande variedade de nutrientes presentes. A quantidade de proteínas nos seus produtos é considerada de alta ou boa qualidade, estudos indicam o perfil de aminoácidos dessa cepa possui todos os aminoácidos essenciais para humanos (arginina, isoleucina, leucina, lisina, metionina, fenilalanina, treonina, triptofano, valina e histidina). Algumas de suas espécies podem possuir até $65 \%$ da sua composição de carboidratos como fibras alimentares. Sua composição de óleo se destaca por possuir ácido $\alpha$ linolênico e ácido linoléico, possuindo aproximadamente 65-70\% do total de ácidos graxos encontrados como ácidos graxos poli-insaturados. Em relação as vitaminas, possui todas as necessárias por humanos, ou seja, B1, B2, B6, B12, niacina, folato, biotina, ácido pantotênico, C, D2, E e K, e $\alpha$ - e $\beta$-carotenos (BITO et al., 2020).

Tanto a Spirulina quanto a Chlorella tem sido comercializada com apelos de superalimento ou comida do futuro. Diversas atividades são relatadas e em parte confirmadas por diferentes tipos de experimentos e ensaios clínicos. Dessa 
forma, essas atividades são dependentes das composições químicas das biomassas, consequentemente dependem de diferentes condições de cultivo, como temperatura, composição de nutrientes e disponibilidade de luz, que podem alterar os níveis de macro e micronutrientes e outros bioativos, como antioxidantes, presentes na biomassa (BITO et al., 2020; NICOLETTI, 2016).

Além do elevado potencial para suplementação alimentar, as algas também apresentam elevado potencial de utilização na indústria cosmética (FONSECA, 2016). Nestas aplicações podem utilizar a denominações de fitocosméticos, por serem naturais e/ou orgânicos. Os ativos podem ser apresentados na forma de um extrato, óleo ou óleo essencial, e correspondem a um segmento da ciência cosmetológica que se dedica ao estudo e à aplicação dos extratos e princípios ativos obtidos dos vegetais em proveito da higiene, estética, correção e manutenção de um estado normal e sadio da pele (SIMÃO, 2019).

A aplicação dos extratos de microalgas nesse setor industrial não é recente e já se encontram no mercado diversos produtos derivados principalmente de Chlorella vulgaris e Spirulina sp. Na cosmética, a aplicação das algas está muito direcionada principalmente para o tratamento cutâneo, com distintas apresentações no mercado, como cremes antienvelhecimento, regeneradores, anti-irritantes, adelgaçantes, antirrugas, preventores de estrias, proliferadores celulares e esfoliantes. Alguns de seus compostos possuem um mecanismo de ação que estimula a síntese de colágeno da pele, trazendo mais flexibilidade e, assim, reduzindo as rugas. Também existem protetores solares para a pele ou para o cabelo que usam extratos de algas para proteção contra as radiações UVA e UVB. Os fitocosméticos que contêm microalgas ou seus extratos estão em pleno crescimento, especialmente quando combinado com outros antioxidantes ou bioativos, para proteger a pele (FONSECA, 2016; VASCONCELOS et al., 2010; WANG et al., 2017).

As microalgas ainda apresentam compostos fotoprotetores, como os aminoácidos tipo micro porinas (chinorina, palatina, asterina e palitinol), que podem ser aplicados na produção de bloqueadores solares, além de compostos antioxidantes que protegem a pele contra as agressões das radiações solares (FONSECA, 2016). Além desses compostos, outro em destaque é a astaxantina, que se classifica como um carotenoide, e protege as células da foto-oxidação. 
As algas expostas às radiações ultravioletas produzirão compostos de triagem ultravioleta, como os aminoácidos semelhantes à micro porina (MAA), que atuaram como antioxidantes e envolvidos nas regulações osmóticas. Além disso, algas expostas à alta radiação solar e baixa concentração de nitrogênio produzem mais carotenos, como Dunaliella. Assim, algas naturalmente expostas ao estresse oxidativo desenvolvem sistemas de defesa que as protegem contra espécies reativas de oxigênio (ROS) e radicais livres. Esses compostos podem ser usados em cosméticos para proteger as células contra os efeitos adversos da radiação UV (THIYAGARASAIYAR et al., 2020). Outro carotenoide que tem sido associado a capacidade de neutralizar o estresse oxidativo causado pela radiação UV é a fucoxantina, sendo, portanto, aplicável em fitocosméticos (WANG et al., 2015).

Os pigmentos microalgais, além da indústria cosmética encontraram mercado também na indústria de alimentos como corantes e, se destacaram em relação aos pigmentos sintéticos por serem atóxicos e não cancerígenos. Como os humanos não os sintetizam, os pigmentos de microalgas têm sido empregados tanto como corantes naturais quanto como suplementos alimentares (VENDRUSCOLO et al., 2020). Estudos evidenciaram que a astaxantina pode suprimir a hiperpigmentação da pele, através da inibição da síntese de melanina, e melhorar o estado de todas as camadas da pele, quer através da sua aplicação tópica, quer oral (FONSECA, 2016).

É provável que esses organismos sejam capazes de abastecer a indústria de cosméticos com ambas as ficobilinas e carotenoides, que significa que uma ampla gama de cores azul, amarelo, laranja e vermelho será coberta. Os tetras pirróis chamados ficobilinas representam os principais pigmentos acessórios fotossintéticos das cianobactérias e algas eucarióticas pertencentes à grupos Glaucófita, Cristófita e Rodófita. Esses pigmentos são proteínas ligadas covalentemente, chamadas ficobiliproteínas, que são, em geral, organizados em ficobilissomos nas membranas tilacóides. Várias espécies de cianobactérias foram relatadas por serem fontes dessa ficocianina, como Arthospira platensis (Spirulina), Arthrospira maxima, Pyrophyridium sp. e Synechocystis (COTEAU e COIFFARD, 2020). 


\section{Perspectivas e aplicações futuras}

A aplicação das microalgas na indústria vai além de compostos nutracêuticos e suplementos alimentares. Dianursanti et al., 2020 estudaram a Spirulina como substituinte do colágeno para matéria prima do invólucro de cápsulas, devido a presença de polihidroxibutirato (PHB) em sua composição. Este polímero atua como agente de liberação de fármacos e prolonga a dissolução dos fármacos, melhorando o desempenho do fármaco na hora da liberação no intestino. Os resultados mostraram-se promissores, a cápsula feita com 3\% (p/v) de concentração de Spirulina platensis se mantém em condição ácida em pH 1,2 por 75 minutos, com liberação de 61,3\% dos medicamentos, depois em pH 4,5 por 120 segundos e liberação de $54,9 \%$ dos medicamentos e também em pH 6,8 por 240 segundos, liberando $89,6 \%$ dos medicamentos.

Outro campo novo de aplicações para microalgas é a produção de vacinas. Em sua pesquisa, Ramos-Vega et al. (2021) selecionaram antígenos com base em seus efeitos protetores através de estudos realizados com ferramentas in-silico. O projeto foi focado no tipo de antígeno e sua localização celular. Devido as novas e várias ferramentas de engenharia genética desenvolvidas, as células microalgais como pró-hosts de produção têm várias vantagens, assim, essa plataforma pode ser usada para purificar antígenos para formulações injetáveis ou como um hospedeiro de entrega para administração mucosa. A última abordagem é útil, uma vez que os antígenos podem ser encapsulados, evitando a cadeia de frio. Hoje em dia, vários antígenos têm sido produzidos em uma grande quantidade de espécies microalgais contra doenças infecciosas para animais e humanos. Em humanos, antígenos promissores têm sido pesquisados em microalgas para combater doenças causadas pelo Vírus da imunodeficiência humana, vírus do papiloma humano, Influenza, hepatite B, Ebola, Zika vírus, Staphylococcus aureus e Plasmodium falciparum. Em animais, os protótipos de vacinas feitas com microalgas foram direcionados para combater os vírus da peste suína clássica, vírus da febre aftosa, vírus da síndrome da mancha branca, vírus da doença Gumboro, Vibriose e Histophilus somni. Em comparação com outras espécies de microalgas, a espécie Chlamydomonas reinhardtii tem sido a mais utilizada, uma vez que já existem as ferramentas de engenharia biotecnológica necessárias para a produção das vacinas. No entanto, cada espécie de microalga tem características atraentes 
que devem ser pesquisadas para serem introduzidas nesse setor. Os resultados da pesquisa têm demonstrado que as microalgas podem produzir antígenos com dobramento adequado e níveis suficientes de rendimento para se tornarem imunogênicas em pré-estudos clínicos. Apesar dos esforços feitos na produção de vacinas, os ensaios clínicos estão pendentes para testar a imunogenicidade, segurança e eficácia.

Outro campo em ascensão para aplicabilidade tem se destacado pela inovação, é para a regeneração de pele. Devido a composição da biomassa microalgal, as mesmas vêm sendo aplicadas em estudos associados a feridas cutâneas e demonstrado efeitos positivos frente a rápida cicatrização. Conforme destacado no estudo publicado por Miguel et al. (2021), os potenciais efeitos benéficos dos carotenoides, ácidos graxos poli-insaturados, polissacarídeos foram relatados em aplicações de cicatrização de feridas devido às suas diferentes atividades biológicas. Os extratos realizados com o gênero de microalgas verdes, de Chlorella, Chlamydomonas, Tetraselmis, Haematococcus e Nannochloropsis são os mais comumente usados para regeneração da pele. Os autores observaram ainda que a presença de compostos de microalgas em formulações curativas diminui os processos de estresse oxidativo, reduz a inflamação e infiltração de células pró-inflamatórias e secreção de citocinas, evitando a colonização do microrganismo no local da ferida, que é de extrema importância para melhorar o processo de cicatrização de feridas.

Os autores Fagundes et al. (2021) observaram o potencial do extrato rico em fitoesteróis atuar como neuroprotetor, devido a inibição da enzima acetilcolina esterase, uma das enzimas chave na doença de Alzheimer, neste mesmo trabalho o extrato foi responsável por inibir também a lipoxigenase, demonstrando ter efeito também de redução do processo inflamatório.

\section{Desafios na aplicabilidade}

Os desafios na aplicação de compostos bioativos de algas estão relacionados com as formas de extração de componentes funcionais, devido ao custo elevado de energia e produção, muitas vezes sendo necessário a aplicação de tecnologias emergentes para o processo extrativo, como exemplo: o uso de extrações assistidas por ultrassom, extrações com micro-ondas ou líquidos pressurizados, entre outros. Nesse sentido, surge a necessidade de 
desenvolver processos com baixo custo, tais problemas podem ser superados através de estudos científicos para novos processos visando sempre uma melhor viabilidade econômica (JHA et al., 2017; SAVIO et al., 2021).

Logo, a falta de informação sobre a ecologia, fisiologia e os diferentes cultivos microalgais é um fator crítico, principalmente quando não há informações concretas sobre o metabólito responsável pela bioatividade observada para novos medicamentos e devido à falta de informações sobre os genomas e mecanismo de regulação gênica de compostos bioativos em algas, ainda existem grandes restrições e limitações que impedem o desenvolvimento deste tipo de pesquisa (SAVIO et al., 2021; WANG et al., 2017).

\section{Referências}

ABIDIZADEGAN, Maryam; PELTOMAA, Elina; BLOMSTER, Jaanika. The potential of cryptophyte algae in biomedical and pharmaceutical applications. Frontiers in Pharmacology, v. 11, 2020.

APONE, Fabio; BARBULOVA, Ani; COLUCCI, Maria Gabriella. Plant and microalgae derived peptides are advantageously employed as bioactive compounds in cosmetics. Frontiers in plant science, v. 10, p. 756, 2019.

BARONI, Luciana et al. Effect of a Klamath algae product ("AFA-B12") on blood levels of vitamin B12 and homocysteine in vegan subjects: a pilot study. International journal for vitamin and nutrition research, v. 79, n. 2, p. 117123, 2009.

BILAL, Muhammad et al. High-value compounds from microalgae with industrial exploitability-A review. Front Biosci, v. 9, n. 3, p. 319-342, 2017.

BITO, Tomohiro et al. Potential of Chlorella as a Dietary supplement to promote human health. Nutrients, v. 12, n. 9, p. 2524, 2020.

BULE, Mohammed Hussen et al. Microalgae as a source of high-value bioactive compounds. Front. Biosci, v. 10, p. 197-216, 2018.

COUTEAU, Céline; COIFFARD, Laurence. Phycocosmetics and other marine cosmetics, specific cosmetics formulated using marine resources. Marine drugs, v. 18, n. 6, p. $322,2020$. 
DA SILVA VAZ, Bruna et al. Microalgae as a new source of bioactive compounds in food supplements. Current Opinion in Food Science, v. 7, p. 73-77, 2016.

DANTAS, Danielli Matias de Macêdo. Atividade biológicas das preparações obtidas das clorofíceas Chlorella vulgaris e Scenedesmus subspicatus Chodate suas potenciais aplicações biotecnológicas. 2013. Tese (Doutorado em Ciências Biológicas) - Universidade Federal de Pernambuco.

DERNER, Roberto Bianchini et al. Microalgas, produtos e aplicações. Ciência Rural, v. 36, p. 1959-1967, 2006.

DIANURSANTI; SARI, Putri R.; ALIFIA, Kanya CH. Utilization of microalgae Spirulina platensis as a raw material for making capsule shell. In: AIP Conference Proceedings. AIP Publishing LLC, 2020. p. 040025.

DUFOSSÉ, Laurent. Current and potential natural pigments from microorganisms (bacteria, yeasts, fungi, microalgae). In: Handbook on Natural Pigments in Food and Beverages. Woodhead Publishing, 2016. p. 337-354.

FAGUNDES, Mariane Bittencourt et al. Insights in cyanobacteria lipidomics: A sterols characterization from Phormidium autumnale biomass in heterotrophic cultivation. Food Research International, v. 119, p. 777-784, 2019.

FAGUNDES, Mariane Bittencourt et al. Green microsaponification-based method for gas chromatography determination of sterol and squalene in cyanobacterial biomass. Talanta, v. 224, p. 121793, 2021.

FAGUNDES, Mariane Bittencourt et al. Phytosterol-rich compressed fluids extracts from Phormidium autumnale cyanobacteria with neuroprotective potential. Algal Research, v. 55, p. 102264, 2021.

FONSECA, Juliana Azevedo. Aplicação de Algas na Indústria Alimentar e. Farmacêutica. 2016. 63 f. Dissertação (Mestrado) - Curso de Ciências Farmacêuticas, Universidade Fernando Pessoa, Porto.

FORZZA, RC., org., et al. Instituto de Pesquisas Jardim Botânico do Rio de Janeiro. Catálogo de plantas e fungos do Brasil [online]. Rio de Janeiro: Andrea Jakobsson Estúdio: Instituto de Pesquisa Jardim Botânico do Rio de Janeiro, 2010. 871 p. Vol. 1. 
FU, Weiqi et al. Bioactive compounds from microalgae: Current development and prospects. In: Studies in natural products chemistry. Elsevier, 2017. p. 199-225.

GAGO, Alexandre da Silva. Compostos bioativos de microalgas com interesse no tratamento da diabetes. 2016. Dissertação (Mestrado em Biologia Molecular e Microbiana) - Faculdade de Ciências e Tecnologia, Universidade do Algarve GARCíA, José L.; DE VICENTE, Marta; GALÁN, Beatriz. Microalgae, old sustainable food and fashion nutraceuticals. Microbial biotechnology, v. 10, n. 5, p. 1017-1024, 2017.

GROSSHAGAUER, Silke; KRAEMER, Klaus; SOMOZA, Veronika. The true value of Spirulina. Journal of agricultural and food chemistry, v. 68, n. 14, p. 4109-4115, 2020.

JACOB-LOPES, Eduardo et al. Bioactive food compounds from microalgae: An innovative framework on industrial biorefineries. Current Opinion in Food Science, v. 25, p. 1-7, 2019.

JHA, Durga et al. Microalgae-based Pharmaceuticals and Nutraceuticals: An Emerging Field with Immense Market Potential. ChemBioEng Reviews, v. 4, n. 4, p. 257-272, 2017.

$\mathrm{KIM}$, Ji Hye et al. Beneficial effects of marine algae-derived carbohydrates for skin health. Marine drugs, v. 16, n. 11, p. 459, 2018.

KUMAR, B. Ramesh et al. Microalgae as rich source of polyunsaturated fatty acids. Biocatalysis and agricultural biotechnology, v. 17, p. 583-588, 2019.

LUO, Xuan; SU, Peng; ZHANG, Wei. Advances in microalgae-derived phytosterols for functional food and pharmaceutical applications. Marine drugs, v. 13, n. 7 , p. $4231-4254,2015$.

MIGUEL, Sónia P. et al. Application of microalgae and microalgal bioactive compounds in skin regeneration. Algal Research, v. 58, p. 102395, 2021.

MOROCHO-JÁCOME, Ana Lucía et al. (Bio) Technological aspects of microalgae pigments for cosmetics. Applied Microbiology and Biotechnology, p. 1-10, 2020. 
MOSHOOD, Taofeeq D.; NAWANIR, Gusman; MAHMUD, Fatimah. Microalgae biofuels production: A systematic review on socioeconomic prospects of microalgae biofuels and policy implications. Environmental Challenges, p. 100207, 2021.

NICOLETTI, Marcello. Microalgae nutraceuticals. Foods, v. 5, n. 3, p. 54, 2016.

PANGESTUTI, Ratih; SIAHAAN, Evi Amelia; KIM, Se-Kwon. Photoprotective substances derived from marine algae. Marine drugs, v. 16, n. 11, p. 399, 2018. PEDROSA, Gabriela Costa et al. Alimentos funcionais: legislação, comparações e um olhar sobre o café. 2015. Monografia (Especialização em Farmacologia) Curso de Ciências Farmacêuticas, Universidade Federal de Minas Gerais PÉREZ, Jessy Pavón et al. Current analytical techniques for the characterization of lipophilic bioactive compounds from microalgae extracts. Biomass and Bioenergy, v. 149, p. 106078, 2021.

PIMENTEL, Carolina Vieira de Mello Barros; ELIAS, Maria Fernanda; PHILIPPI, Sonia Tucunduva. Alimentos funcionais e compostos bioativos. [S.I: s.n.], 2019.

RAMOS-VEGA, Abel et al. Microalgae-made vaccines against infectious diseases. Algal Research, v. 58, p. 102408, 2021.

RANDHIR, Ankitha et al. Microalgae: a potential sustainable commercial source of sterols. Algal Research, v. 46, p. 101772, 2020.

RIBEIRO, Michele Caldeira Magdalena. Produção de biofármacos por microalgas: mapeamento tecnológico, avaliação e caracterização de extratos com efeito antiviral sobre o vírus Mayaro. 2019. Dissertação (Mestrado em Engenharia de Processos Químicos e Bioquímicos) - Universidade Federal do Rio de Janeiro.

RIGHI, Valeria et al. Mycosporine-like amino acids and other phytochemicals directly detected by high-resolution NMR on Klamath (Aphanizomenon flosaquae) blue-green algae. Journal of agricultural and food chemistry, v. 64, n. 35, p. 6708-6715, 2016. 
SAIDE, Assunta et al. Unlocking the Health Potential of Microalgae as Sustainable Sources of Bioactive Compounds. International Journal of Molecular Sciences, v. 22, n. 9, p. 4383, 2021.

SAMI, Neha; AHMAD, Rakhshan; FATMA, Tasneem. Exploring algae and cyanobacteria as a promising natural source of antiviral drug against SARS-CoV2. Biomedical Journal, 2020.

SATHASIVAM, Ramaraj et al. Microalgae metabolites: A rich source for food and medicine. Saudi journal of biological sciences, v. 26, n. 4, p. 709-722, 2019.

SAVIO, Saverio; CONGESTRI, Roberta; RODOLFO, Carlo. Are we out of the infancy of microalgae-based drug discovery?. Algal Research, v. 54, p. 102173, 2021.

SIMÕES, Mirela Assunção et al. Algas cultiváveis e sua aplicação biotecnológica (recurso eletrônico). Aracaju: IFS, 2016.

SINGH, Shailendra Kumar et al. Biotechnological exploitation of cyanobacteria and microalgae for bioactive compounds. In: Biotechnological Production of Bioactive Compounds. Elsevier, 2020. p. 221-259.

TANG, Doris Ying Ying et al. Potential utilization of bioproducts from microalgae for the quality enhancement of natural products. Bioresource technology, v. 304, p. 122997, 2020.

THIYAGARASAIYAR, Krishnapriya et al. Algae metabolites in cosmeceutical: An overview of current applications and challenges. Marine drugs, v. 18, n. 6, p. 323, 2020.

VIEIRA, Marta V.; PASTRANA, Lorenzo M.; FUCIÑOS, Pablo. Microalgae Encapsulation Systems for Food, Pharmaceutical and Cosmetics Applications. Marine drugs, v. 18, n. 12, p. 644, 2020.

WANG, Hui-Min David et al. Exploring the potential of using algae in cosmetics. Bioresource technology, v. 184, p. 355-362, 2015.

WANG, Hui-Min David et al. Potential biomedical applications of marine algae. Bioresource technology, v. 244, p. 1407-1415, 2017. 


\section{Autores}

Juliana Mesadri*, Roger Wagner, Mariane Bittencourt Fagundes.

Departamento de Tecnologia e Ciência dos Alimentos, Centro de Ciências Rurais, Universidade Federal de Santa Maria Endereço: Av. Roraima, 1000, CEP 97105-900, Santa Maria/RS, Brasil.

*Autor para correspondência: juliana_mesadri@hotmail.com 